\title{
FACTORES OBSTÉTRICOS IMPLICADOS EN LA APARICIÓN DE INFECCIÓN DE SITIO QUIRÚRGICO EN MUJERES SOMETIDAS A CESÁREA
}

\author{
Eliana Betsy Cabrera Palomino1,a
}

\begin{abstract}
RESUMEN
Objetivos. Identificar los factores obstétricos implicados en el desarrollo de una infección en sitio quirúrgico (ISQ) en pacientes sometidas a cesárea en el Instituto Nacional materno Perinatal. Materiales y métodos. es un estudio transversal, descriptivo, retrospectivo. Se enrolaron 85 pacientes que presentaron ISQ. Se analizaron las características sociodemográficas y ginecoobstétricas, factores de riesgo condicionantes. Se utilizó una ficha de recolección de datos y se procesó con SPS V20.2. Resultados. Edad prevalente entre 20-35 años, primíparas 63.5\%, atención prenatal insuficiente $55 \%$, obesidad $47 \%$, cesárea de emergencia fue la más común con $88.2 \%$, la principal indicación de cesárea fue mala actitud de presentación $14 \%$, tiempo operatorio< 45 min fue mayoritario $51.8 \%$. Tuvieron RPM en $22 \%$ y anemia leve en $48 \%$. Conclusiones. Los principales factores condicionantes para ISQ fueron cesárea de emergencia, atención prenatal insuficiente, ruptura prematura de membranas y obesidad materna.
\end{abstract}

Palabras claves: Infección de sitio quirúrgico, poscesárea, obesidad materna (Fuente: DeCS BIREME).

\section{OBSTETRIC FACTORS INVOLVED IN THE APPEARANCE OF SURGICAL SITE INFECTION IN WOMEN UNDERGOING CAESAREAN SECTION}

\begin{abstract}
Objectives. To identify the clinical characteristics that influence the development of a surgical site infection (ISQ) in patients undergoing caesarean section in the National Maternal Perinatal Institute. Materials and methods. it is a cross-sectional, descriptive, retrospective study. 85 patients who presented ISQ were enrolled. The sociodemographic and gyneco-obstetric characteristics, conditioning risk factors were analyzed. A data collection sheet was used and processed with SPS V20.2. Results. Prevalent age between 20-35 years, primiparous 63.5\%, insufficient prenatal care $55 \%$, obesity $47 \%$, emergency caesarean section was the most common with $88.2 \%$, the main indication for caesarean section was bad presentation attitude $14 \%$, operating time $<45$ min The majority was $51.8 \%$. They had RPM in $22 \%$ and mild anemia in $48 \%$. Conclusions. The main conditioning factors for SSI were emergency caesarean section, insufficient prenatal care, premature rupture of membranes and maternal obesity.
\end{abstract}

Keywords: Surgical site infection, post-caesarean, maternal obesity (Source: MeSH NLM).

\section{INTRODUCCIÓN}

La cesárea es una de las cirugías en obstetricia que se practican con más frecuencia a nivel global y nacional. La complicación más frecuente de dicho procedimiento es la infección de sitio quirúrgico (ISQ) que a pesar de la tendencia a la disminución en los tiempos actuales, aún constituye el principal problema de las infecciones asociadas a la salud que ocasionan estancias prolongadas y mayores costos hospitalarios.

La ISQ es una de las causas más frecuentes de infección hospitalaria, ocupando el segundo lugar en la mayoría de los estudios de infecciones nosocomiales, y la primera entre los pacientes quirúrgicos ${ }^{1}$. Donde su incidencia varía de un cirujano a otro, de hospital en hospital, de un procedimiento quirúrgico a otro, y más importante de un paciente a otro ${ }^{1,2}$.

La ISQ pertenece a la lista de las infecciones nosocomiales y ocurre en 3 a $16 \%$ de las operaciones cesáreas ${ }^{3}$.

Se ha informado que la tasa de infección después de un parto por cesárea es del 4 al $15 \%{ }^{4-6}$ aunque los datos recientes de NHSN mostró una tasa de infección del 2-4\%.

Cabe señalar que las infecciones del sitio quirúrgico son causadas por bacterias que ingresan a través de incisiones hechas durante la cirugía. Amenazan la vida de millones de pacientes cada año y contribuyen a la propagación de la resistencia a los antibióticos. En países de bajos y medianos ingresos, el $11 \%$ de los pacientes que

\footnotetext{
1 Instituto Nacional Materno Perinatal.

a Médico Cirujano, Asistente de Unidad de Referencias de Emergencia.

Citar como: Cabrera Palomino EB Factores obstétricos implicados en la aparición de infección de sitio quirúrgico en mujeres sometidas a cesárea. Rev Peru Investig Matern Perinat. 2019; 8(3):17-20 DOI https://doi.org/10.33421/inmp.2019160
}

Recibido: 07-08-2019 Aceptado: 27-09-2019 
se someten a cirugía están infectados en el proceso. En África, hasta el $20 \%$ de las mujeres que tienen una cesárea contraen una infección de la herida, comprometiendo su propia salud y su capacidad para cuidar a sus bebés ${ }^{7}$.

Es necesario resaltar que se consideran como factores de riesgo para infección de la herida quirúrgica que se irán desarrollando progresivamente: a)Dependientes del paciente: estado inmunitario, enfermedades de base, hábitos nocivos, tratamientos habituales, estado nutricional, infecciones coexistentes; b) Dependientes del acto quirúrgico: técnica quirúrgica, duración de la cirugía, localización y tipo de cirugía, uso de profilaxis antibiótica, asepsia y antisepsia del quirófano, personal e instrumental utilizado; y c) Otros: cuidados postoperatorios, duración de la estancia hospitalaria prequirúrgica, funcionamiento de los drenajes ${ }^{8}$.

Asimismo, múltiples factores se han asociado con un mayor riesgo de infección del sitio quirúrgico, incluido la obesidad, hábito de fumar, abuso de alcohol, edad avanzada, hospitalización preoperatoria prolongada, diabetes, glucosa sérica elevada (>125 mg / dL antes de la operación [dentro de 30 días] o >200 mg / dL después de la operación) y procedimiento previo complicado por infección ${ }^{4}$.

La cesárea está considerada como un procedimiento contaminado cuando existe trabajo de parto previo o ruptura prematura de membranas y aséptico cuando estos dos eventos no se presentan ${ }^{9}$.

La infección del sitio quirúrgico (ISQ) después del parto por cesárea es una causa importante de morbilidad y mortalidad, aumentando tanto la duración de la hospitalización del paciente y los costos hospitalarios ${ }^{1,2,9}$.

En el Instituto Nacional Materno Perinatal (INMP) de Lima, durante el periodo enero a diciembre 2018 ocurrieron 17,754 partos, de los cuales el $43 \%$ (7,643 casos) fueron parto por cesárea. Asimismo, durante el mismo periodo se registró 4,494 casos infecciones maternas que representan el $25,3 \%$ de los egresos hospitalarios, de los cuales corresponden a infección de herida operatoria 131 casos que equivale a $0,7 \%$ del total de infecciones maternas ${ }^{10}$.

Mientras que en otros estudios la tasa de incidencia de la ISQ después de cesárea la sitúa en un rango de $3 \%$ a $5 \%$, variando de acuerdo a la población objeto de estudio, los métodos utilizados para monitorear e identificar los casos, y el uso de la profilaxis con antibióticos apropiados ${ }^{11-13}$.

Asimismo, en el INMP la tasa de incidencia acumulada durante el periodo 2018 en ginecoobstetricia corresponde a $1,3 \times 100$ personas expuestas (PE) y su tendencia en los últimos 9 años ha sido descendiente con tasas que varían desde 2.8 a 1.45 x 100 PE entre los años 2009- 2014 ${ }^{14}$.

Además la infección de sitio quirúrgico por cesárea constituye el $35 \%$ del total de Infecciones asociadas a la atención en salud (IASS) antiguamente llamadas Infecciones intrahospitalarias (IIH) seguido por la Infección de torrente sanguíneo debido a catéter venoso periférico en un $33,2 \%$ y en un tercer lugar a la endometritis postcesárea en $10,8 \%{ }^{14}$. Por lo tanto la ISQ debido a la cesárea constituye la causa más importante de morbilidad en cuanto las IASS, de ahí radica su importancia por conocer sus factores y evitar su presentación.

\section{MATERIALES Y MÉTODOS}

Fue un estudio observacional, descriptivo, retrospectivo y de corte transversal, que se realizó en el Instituto Nacional Materno Perinatal INMP) de Lima durante el periodo de enero a diciembre de 2015. La población de estudio fueron mujeres en las que se diagnosticó una infección de sitio quirúrgico (ISQ) luego de haber sido sometidas a cesárea. La muestra fue constituida por 85 mujeres que cumplieron con los criterios de inclusión. Las variables del estudio fueron edad materna, paridad, tipo de ISQ, índice de masa corporal, indicación de cesárea, duración del tiempo quirúrgico, factores obstétricos como ruptura prematura de membranas y anemia. Todos los datos recolectados a partir de las historias clínicos fueron recogidos en una ficha estructurada. El procedimiento consistió en presentar una solicitud a la Dirección General del INMP para su aprobación, luego la oficina de estadística e informática remitió un listado con los números de las historias clínicas de las mujeres que salieron con el diagnóstico de infección de sitio quirúrgico durante el 2015. Del total de 115 historias clínicas con el diagnóstico de ISQ al egreso, 7 no se pudieron hallar, 11 no tenían el diagnóstico de ISQ, en 5 mujeres la operación no había sucedido en la institución y finalmente 7 no tuvieron datos suficientes para completar la ficha de datos. Durante 5 días se completó el total de fichas que fueron 85 , todos los datos se recopilaron en Excel 2007, para luego ser procesados por el programa estadístico SPSS20.2 y así obtener los porcentajes y las tablas correspondientes. Se respetó la confidencialidad de los datos obtenidos de las historias clínicas. La presente investigación no requirió consentimiento informado.

\section{RESULTADOS}

En la tabla 1 se evidencia que $89,4 \%$ de mujeres tuvieron una ISQ superficial, $9,4 \%$ ISQ profunda y $1,2 \%$ ISQ de órgano-espacio.

\section{Tabla 1: Tipos de ISQ}

\begin{tabular}{lcc}
\hline \multicolumn{1}{c}{ Tipos ISQ } & N & \% \\
\hline Superficial & 76 & 89,4 \\
Profunda & 8 & 9,4 \\
Espacio - Órgano & 1 & 1,2 \\
Total & 85 & 100 \\
\hline
\end{tabular}


Tabla 2. Características obstétricas en ISQ.

\begin{tabular}{|c|c|c|}
\hline Características obstétricas de ISQ & $\mathbf{N}$ & $\%$ \\
\hline \multicolumn{3}{|l|}{ Edad materna (años) } \\
\hline \multicolumn{3}{|l|}{$<19$} \\
\hline $19-34$ & 55 & 64,8 \\
\hline 35 a más & 15 & 17,6 \\
\hline \multicolumn{3}{|l|}{ Paridad } \\
\hline Primípara & 54 & 63,5 \\
\hline Multípara & 29 & 34,1 \\
\hline Gran multípara & 2 & 2,4 \\
\hline \multicolumn{3}{|l|}{ Presencia de Obesidad } \\
\hline Sí & 40 & 47.05 \\
\hline No & 45 & 52.9 \\
\hline \multicolumn{3}{|l|}{ Ruptura prematura de membranas } \\
\hline$<6 \mathrm{~h}$ & 3 & 3,5 \\
\hline $6-12 \mathrm{~h}$ & 3 & 3,5 \\
\hline $12-24 \mathrm{~h}$ & 1 & 1,2 \\
\hline$>24 \mathrm{~h}$ & 11 & 12,9 \\
\hline No & 67 & 78,8 \\
\hline \multicolumn{3}{|l|}{ Sometidas a cesárea de emergencia } \\
\hline $\mathrm{Si}$ & 75 & 88,2 \\
\hline No & 10 & 11,8 \\
\hline \multicolumn{3}{|l|}{ Tiempo de duración de cesárea } \\
\hline$<45 \min$ & 44 & 51,8 \\
\hline$>45 \min$ & 41 & 48,2 \\
\hline \multicolumn{3}{|l|}{ Clasificación de anemia postcesárea } \\
\hline Ninguna & 16 & 18,8 \\
\hline Anemia leve & 41 & 48,2 \\
\hline Anemia moderada & 21 & 24,7 \\
\hline Anemia severa & 7 & 8,2 \\
\hline
\end{tabular}

Según la tabla 2 en todas las edades reproductivas se presentaron ISQ, con una prevalencia importante en ambos extremos de la vida; en la mayoría se presentaron ISQ en las primíparas $(63,5 \%)$; la presencia de obesidad ocupó el $47,05 \%$. La ruptura prematura de membranas mayor a 24 horas ocupó el 12,9\% de los casos estudiados. La mayoría de los casos de ISQ fueron sometidos a cesárea de emergencia (88,2\%). El tiempo de duración de la cesárea estuvo dentro de los 45 minutos en promedio. En relación a la hemoglobina de control posoperatorio revela que el $48,2 \%$ de casos presentaron anemia leve y el $8,2 \%$ anemia severa.

Según la tabla 3, la indicación de cesárea predominante fue mala actitud de presentación $14,1 \%$, seguida por sufrimiento fetal agudo con $9.4 \%$, cesareada anterior 2 veces 8,2\%, 7,1\% mujeres por incompatibilidad cefalopélvica, al igual que insuficiencia placentaria, presentación podálica, macrosomía fetal y cesareada anterior 1 vez.
Tabla 3. Indicación de la cesárea

\begin{tabular}{lcc}
\hline \multicolumn{1}{c}{ Diagnóstico } & N & $\%$ \\
\hline Arritmia fetal & 1 & 1,2 \\
\hline Cesareada anterior 1 vez & 6 & 7,1 \\
\hline Cesareada anterior 2 veces & 7 & 8,2 \\
\hline Cesareada anterior 3 veces & 2 & 2,4 \\
\hline Corioamnionitis & 2 & 2,4 \\
\hline Desprendimiento de retina & 1 & 1,2 \\
\hline Distocia funicular & 5 & 5,9 \\
\hline Desprendimiento Prematura Placenta & 1 & 1,2 \\
\hline Eclampsia & 1 & 1,2 \\
\hline Feto con gastrosquisis & 1 & 1,2 \\
\hline Incompatibilidad cefalopélvica & 6 & 7,1 \\
\hline Insuficiencia placentaria & 6 & 7,1 \\
\hline Macrosomía fetal & 6 & 7,1 \\
\hline Mala actitud de presentación & 12 & 14,1 \\
\hline Placenta previa & 5 & 5,9 \\
\hline Podálico & 6 & 7,1 \\
\hline Preeclampsia severa & 5 & 5,9 \\
\hline Prolapso de cordón & 2 & 2,4 \\
\hline Sufrimiento fetal agudo & 8 & 9,4 \\
\hline Trabajo de parto disfuncional disfuncional & 2 & 2,4 \\
\hline Total & 85 & 100,0 \\
\hline
\end{tabular}

\section{DISCUSIÓN}

De acuerdo al tipo de infección quirúrgica, en nuestro estudio el $89,4 \%$ fue de tipo superficial así como los trabajos de referencia ${ }^{15-17}$ afortunadamente el tipo de ISQ más grave representa un pequeño porcentaje $(1,2 \%)$.

En cuanto a la edad en la que se diagnosticó la ISQ, las mujeres en edad reproductiva(20-35 años) fueron mayoría con un $64,8 \%$; resultados similares presentados por Quiroz $\mathrm{M}^{18}$ y Quinde NR ${ }^{17}$ en el Perú, Jido TA et a/ ${ }^{15} \mathrm{y}$ Franco $\mathrm{FT}$ et a/ ${ }^{19}$ a nivel internacional llama la atención que el porcentaje comparado entre adolescentes y mujeres es el mismo $17,6 \%$, lo cual nos da una idea de que la población en general aplaza su reproductividad respecto a otras metas, así mismo en estudios internacionales este hecho fue corroborado por Aguilar CL et a/ ${ }^{16}$ y Mitt $\mathrm{P}$ et $a{ }^{11}$.

Según la paridad el $63,5 \%$ es primípara situación parecida en el estudio realizado por Quinde NR ${ }^{17}$ y por Mitt $\mathrm{P}$ et al ${ }^{11}$, Jido TA et $a{ }^{15}$ en ámbitos internacionales.

La obesidad en nuestro estudio fue reportada en un $47 \%$, a comparación de hace una década en el estudio de Quiroz $\mathrm{M}^{18}$ realizado en la misma institución. Además, es un factor de riesgo significativo en el desarrollo de la ISQ según Quinde $N R^{17}$. Vemos que a nivel peruano ${ }^{17,18}$ tiene mucha preponderancia, y es alarmante que vaya en 
ascenso siendo un factor en el que se puede prevenir su ausencia.

Si bien la ruptura prematura de membranas sólo se presentó en el $21 \%$ de las cesareadas con ISQ, la más frecuente fue el RPM prolongado con $12 \%$, aunque ha disminuido en la última década ${ }^{18}$. Cabe resaltar que la ruptura prematura de membranas prolongada es actualmente considerada un factor de riesgo significativo corroborado en diversos estudios ${ }^{17,19}$. Esto puede ser debido a que a mayor tiempo de ruptura de membranas, mayor colonización de gérmenes que ascienden por esta vía.

Según lo reportado el $88,2 \%$ de las cesáreas fueron de emergencia mientras que sólo el $11,7 \%$ fue una cesárea electiva. Esta variable ha sido bien estudiada en diversos estudios de casos y controles y llegó a ser considerado un factor de riesgo para el desarrollo de ISQ ${ }^{15,18,19}$.

La causa más frecuente de cesárea en nuestro estudio fue la mala actitud de presentación $14 \%$ y el sufrimiento fetal agudo en $9 \%$, situación parecida al estudio hecho en Estonia por Mitt $\mathrm{P}$ et a $\mathbf{l}^{11}$.La mala actitud de presentación se diagnostica durante de la labor de parto, por lo cual las horas se prolongan y es sabido que mientras más dure un parto, mayor grado de contaminación.

La duración de tiempo operatorio mayor de 45 minutos estuvo presente en un $41 \%$ de las ISQ, y es un factor de riesgo significativo según Quinde $\mathrm{NR}^{17}$ en Perú, Jido TA et $a^{15}$ en Nigeria; mientras que Quiroz $\mathrm{M}^{18}$ no lo considera significativo.

La anemia leve fue mayoritaria en nuestro estudio con un $84 \%$, pero un $24 \%$ tuvo anemia moderada $24 \%$, aparentemente la situación nacional se agravó; pues, los porcentajes presentados por Quiroz $\mathrm{M}^{18}$ fueron casi la mitad de nuestro valor.

En conclusión, el tipo más frecuente de infección de sitio quirúrgico fue la superficial; y los factores prevalentes fueron obesidad, ruptura prematura de membranas mayor a 24 horas y gestantes sometidas a cesárea de emergencia.

Financiamiento: Autofinanciado.

Conflicto de interés: La autora declara no tener algún conflicto de interés.

\section{REFERENCIAS BIBLIOGRÁFICAS}

1. Webster J, Osborne S. Baño o lavado preoperatorio con antisépticos cutáneos para la prevención de la infección del sitio quirúrgico. La Biblioteca Cochrane Plus. 2008(2).

2. Van Kasteren M, Mannien J, Kullberg B, de Boer A, Nagelkerke N, Ridderhof M, et al. Quality improvement of surgical prophylaxis in Dutch hospitals: evaluation of a multi-site intervention by time series analysis. J Antimicrob Chemother. 2005;56(6):1094-102.

3. Zugaib M, Veja CEP, Miyadahira S. Mortes por doenças infecciosas em mulheres: Ocorrências no ciclo gravídicopuerperal. Rev Assoc Med. 2009;55(1):1-11.
4. Bratzler DW, Dellinger EP, Olsen KM, Perl TM, Auwaerter PG, Bolon MK, Weinstein RA. Clinical practice guidelines for antimicrobial prophylaxis in surgery. American Journal of Health-System Pharmacy. 2013; 70(3): 195-283. doi:10.2146/ ajhp120568

5. Martin JA, Hamilton BE, Sutton PD et al. Births: final data for 2006. Natl Vital Stat Rep. 2009; 57:1-104.

6. Edwards JR, Peterson KD, Mu Y et al. National Healthcare Safety Network (NHSN) report: data summary for 2006 through 2008. Am J Infect Control. 2009; 37:783-805.

7. World Health Organization (WHO). Global guideline on prevention of surgical site infection. 2016.

8. Santalla A, López-Criado MS, Ruiz MD, Fernandez-Parra J, Gallo JL, Montoya F. Infección de la herida quirúrgica. Prevención y tratamiento. Clínica e investigación en Ginecología y Obstetricia. 2007; 34(5): 189-196. DOI: 10.1016/S0210573X(07)74505-7

9. Camacho M, Kantu EK, Hilton JM, Mendoza M. Prevención, diagnóstico y tratamiento de la infección en herida quirúrgica post cesárea en los tres niveles de atención. Catálogo Maestro de Guías de Práctica Clínica. México: Secretaría de Salud, 2011:1-33.

10. Instituto Nacional Materno Perinatal. Boletín estadístico 2018. Lima Perú. Disponible en: https://www.inmp.gob.pe/institucional/ boletines-estadisticos/1422371837

11. Mitt $P$, Lang $K$, Peri $A$, Maimets $M$. Surgical-site infectionsfollowing cesarean section in an Estonian university hospital:postdischarge surveillance and analysis of risk factors. InfectControl Hosp Epidemiol. 2005;26:449-54.

12. Schneid-Kofman N, Sheiner E, Levy A, Holcberg G. Risk factors for wound infection following cesarean deliveries. Int JGynaecol Obstet. 2005;90:10-5.

13. Yokoe DS, Noskin GA, Cunningham SM, Zuccotti G, Plaskett T,Fraser VJ. Enhanced identification of postoperative infections.Emerg Infect Dis. 2004;10:1924-30.8.

14. Gonzales O, Infecciones asociadas a la atención en salud (IASS). Oficina de Epidemiología y Salud Ambiental- Instituto Nacional Materno Perinatal. Perú . 2016.

15. Jido TA and Garba ID. Surgical-site Infection Following Cesarean Section in Kano, Nigeria. Ann Med Health Sci Res. 2012 Jan-Jun; 2(1): 33-36. From: http://www.ncbi.nlm.nih. gov/pmc/articles/PMC3507120/

16. Aguilar CL, Vieira FL, Moura BR, De Souza GL, Teixeira VC. Infección de herida operatoria tras cesárea en un hospital público de Fortaleza. Enfermería global. 2013. 12(29). Disponible en http://scielo.isciii.es/scielo.php?pid=S1695 $61412013000100005 \&$ script=sci_arttext.

17. Quinde NR. Factores de riesgo asociados a infección del sitio quirúrgico en pacientes operadas de cesárea atendidas en el Hospital Belén de Trujillo 2012 - 2013. Tesis para optar el título de médico cirujano. Universidad Privada Antenor Orrego. 2014. Perú. 17 pp. Disponible en http://repositorio. upao.edu.pe/handle/upaorep/482.

18. Quiroz M. Infección de herida quirúrgica en cesáreas en el Instituto Materno Perinatal 2002. Tesis para optar el título de Especialista en Gineco- Obstetricia. Lima- Perú. Universidad Nacional Mayor de San Marcos. 2003. 15 pp. Disponible en file:///C:/Users/Eliana/Desktop/TESIS/Quiroz_\%20ISQ\%20 en\%20maternidad\%202003.pdf.

19. Franco FT, Dalle J, Da Silva MV, Werdig RC, Sperb AV. Risk factors for surgical site infection following cesarean section in a Brazilian Women's Hospital: a case-control study. Brazjinfectdis. 2015; 19(2):113-117.

Correspondencia: Eliana Betsy Cabrera Palomino

Dirección: Calle Río Tigre $N^{\circ} 250$, Asociación Villa Hermosa, Distrito El Agustino

Correo: elybs237867@gmail.com

Teléfono: 990906465 